\title{
PRODUCTIVIDAD, COMPETENCIA E INNOVACIÓN EN LA BANCA PRIVADA ESPAÑOLA (1900-1914)
}

\section{XAVIER CUADRAS-MORATÓ Universitat Pompeu Fabra}

ÁNGEL S. FERNÁNDEZ CASTRO

Universidade de A Coruña

\author{
JOAN R. ROSÉS
}

Universidad Carlos III de Madrid

\section{RESUMEN}

Empleando el método DEA para la medición de la productividad y el índice Malmquist, este artículo investiga las diferencias en productividad, y las causas de las mismas, en una muestra de bancos en los últimos años del período «liberal» de la banca española (1900-1914) que se caracterizaron por una serie de cambios cualitativos y cuantitativos que afectaron a todo el sistema financiero. La principal conclusión que obtenemos es que durante el período tuvo lugar un incremento en los niveles de eficiencia en el conjunto del sector resultado del incremento de competición. En segundo lugar, el análisis pone de manifiesto el comportamiento diferencial en términos de productividad de los distintos grupos bancarios regionales que explicaría, al menos parcialmente, su evolución posterior.

\section{ABSTRACT}

Employing DEA productivity analysis and the Malmquist index, this article investigates the productivity differences and its sources across a set of banks during the last years of the liberal era of the Spanish banking system (1900-1914), which was characterised by major qualitative and quantitative changes in the banking industry. We find evidence of an overall increase in efficiency consequence of increasing competition across banks. Also, our esti-

N. de E.: Fecha de recepción del artículo: febrero, 2001.

Fecha de aprobación por el Consejo de Redacción: octubre, 2002. 
mates suggest that differences in productivity could serve to explain, at least in part, the subsequent evolution of the different regional banking groups. JEL Classification No: N23, G2, C1.

\section{INTRODUCCIÓN}

El sistema financiero desempeña un papel determinante en el desarrollo económico de las naciones. Los bancos y otras entidades del sector tienen un lugar esencial tanto en la provisión de liquidez como en la asignación de recursos y, por esa razón, la regulación bancaria tiene un papel central en la política económica de numerosos países. España no constituye una excepción a esta regla, ya que, desde la promulgación de la Ley de Ordenación bancaria en 1921 hasta tiempos muy recientes, el sistema bancario español se caracterizó por un fuerte intervencionismo estatal, la existencia de numerosas barreras de entrada y todo tipo de trabas legales a la competencia entre entidades ${ }^{1}$. Sin embargo, no siempre fue así, ya que, desde 1856 hasta 1920 , el marco legal que afectaba a la banca podría definirse, en palabras de Martín Aceña, como de «liberalismo financiero moderado» ${ }^{2}$.

El objetivo de este estudio es analizar los cambios en la productividad de la banca privada española al final de esta época de liberalismo. En concreto, durante los cruciales años para la construcción del sistema bancario español que discurren entre la derrota en la guerra de Cuba y la I Guerra Mundial. Existen numerosos trabajos que se ocupan del desarrollo de la banca privada durante este período y los anteriores ${ }^{3}$. Sin embargo, a pesar de la amplia bibliografía existente, faltan, a nuestro entender, investigaciones que aborden profundamente un tema muy importante: los efectos que tuvo el marco liberal sobre la evolución de la productividad de los bancos y si ésta tuvo relación con los cambios en la estructura y tamaño del sistema bancario.

Ésta es la cuestión principal que vamos a considerar a lo largo de este trabajo. En otras palabras, el objetivo principal de este artículo es analizar si el incremento de entidades, consecuencia principalmente del marco legal liberal y de la favorable situación económica, que tuvo lugar

'Pons (1999)(2001a)(2001b).

2 Martín Aceña (1985), p. 127.

${ }^{3}$ Véase, entre otros, Anes (1974), Canosa (1945), Martín Aceña (1985), Roldán y García Delgado (1973), Tallada (1946), Tedde de Lorca (1974), Tortella (1970), Tortella y Palafox (1983). 
a principios del siglo $\mathrm{xx}$ significó también un crecimiento de la eficiencia del sector ${ }^{4}$. No es éste, sin embargo, el único asunto que se va a tratar a lo largo de las siguientes páginas, ya que también vamos a analizar las causas que generaron estos cambios en la productividad de la banca privada $y$, en concreto, vamos a tratar de discernir si los incrementos en productividad fueron consecuencia de un proceso de innovación tecnológica o de un aumento generalizado de eficiencia. Asimismo, también trataremos de averiguar si existió una relación causa-efecto entre los incrementos de productividad y el relativo éxito de algunos bancos. En concreto, nos preocupa especialmente la relación que pudiese existir entre el éxito de la banca vasconavarra y el fracaso de la banca catalana y la evolución de la productividad de sus bancos.

\section{UNA ÉPOCA DE GRANDES CAMBIOS EN EL SISTEMA BANCARIO ESPAÑOL}

La masiva repatriación de capitales invertidos en las colonias y la reforma de la hacienda pública del ministro Fernández Villaverde, que equilibró las cuentas del Estado español e inauguró una nueva línea de la política económica más ortodoxa y estable, insuflaron nuevos bríos a la banca privada española a principios del siglo $\mathrm{xx}^{5}$. En 1892 había 35 bancos privados en España con un capital desembolsado de 210 millones de pesetas, mientras que en 1921 la cifra de bancos había alcanzado los 150 y el capital desembolsado llegaba a los 850 millones de pesetas. En el mismo período, los depósitos bancarios crecieron desde 179 millones de pesetas en 1880 a 515 millones de pesetas en $1913^{6}$. Este crecimiento de los depósitos hizo que por primera vez el sistema bancario español dispusiese de más recursos ajenos que propios ${ }^{7}$.

Paralelamente a este proceso de crecimiento, se produjo un proceso generalizado de reordenación y modernización del sistema bancario español

${ }^{4}$ Hemos decidido restringir nuestro análisis al período $1900-1914$, y no seguir con nuestro análisis hasta 1920 , por dos razones principales: la primera es que el análisis del período de la I Guerra Mundial introduce numerosas distorsiones en nuestros resultados a causa de la importante inflación, la llegada de capitales foráneos y los beneficios extraordinarios de algunos sectores y, en segundo lugar, es que los balances de muchos bancos, como el Banco de Barcelona, véase al respecto Muñoz (1988), fueron maquillados durante esa época para ocultar problemas de liquidez y los síntomas de su posterior quiebra.

5 Tedde de Lorca (1974), pp. 341-350.

${ }^{6}$ Martín Aceña (1985), cuadro 6.2, p. 131.

7 Martín Aceña (1985), p. 130. 
que, en 1900, aún era bastante primitivo si lo comparamos con los de los países más avanzados de Europa. En ese año, el imperio del Banco de España sobre el mercado bancario era abrumador, ya que esta entidad por sí sola absorbía el 68 por 100 de los activos totales del sistema. Asimismo, el sistema financiero se encontraba poco diversificado debido a la preponderancia absoluta de la banca privada sobre otros tipos de entidades. Sin embargo, a lo largo de los siguientes treinta años, el dominio del banco emisor se había resquebrajado y las cajas de ahorro y las sociedades oficiales de crédito habían sido capaces de conseguir una parte importante del mercado en detrimento de los bancos privados ${ }^{8}$. Además, las mayores entidades daban los primeros pasos en pos de la construcción de sus redes nacionales de sucursales, tal como las conocemos hoy en día, siendo los pioneros en este proceso el Banco Español de Crédito y el Banco Hispano Americano ${ }^{9}$. Hasta 1900, la conexión entre las diversas plazas se hacía por medio de corresponsalías y sólo el Banco de España, que disponía de la única red nacional de sucursales, y el Banco Hipotecario extendían sus negocios a todo el territorio nacional ${ }^{10}$.

El reparto del mercado bancario entre las diferentes plazas financieras y la organización de los negocios de los bancos también se alteró de forma sustancial durante el período inmediatamente anterior a la I Guerra Mundial. Desde mediados del siglo XIx, Madrid y Barcelona compartían la primacía dentro del mercado financiero español. Después de la crisis de las sociedades de crédito entre 1864 y 1868 y de la concesión del monopolio de emisión al Banco de España en 1874, los bancos comerciales, que mantenían fuertes lazos con las actividades de las bolsas locales, predominaron en ambas plazas. Sin embargo, a finales del siglo $\mathrm{xx}$, el fulgurante ascenso de los bancos del Norte modificó dramáticamente la anterior situación. En primer lugar, porque los bancos vascos aumentaron su cuota de mercado pasando de gestionar el 6,62 por 100 de los activos del sector en 1874 al 43,65 por 100 en 1913. Por tanto, Bilbao pasó a ser el núcleo principal de la banca privada española. En segundo lugar, porque los bancos con sede en Bilbao actuaban en el mercado de manera diferente a como lo habían hecho, hasta entonces, los bancos catalanes y madrileños. En concreto, tenían una notable preferencia por las actividades de la banca «mixta»; es decir, combinaban las actividades comerciales e intermediarias con la participación directa en sociedades industriales en las que sus directivos

\footnotetext{
${ }^{8}$ Martín Aceña (1985), cuadro 6.1, p. 129.

9 Tedde de Lorca (1974), p. 351.

${ }^{10}$ Castañeda y Tafunell (1993).
} 
ocupaban asientos en los consejos de administración. Este proceder de los bancos vascos fue imitado por el resto de grandes entidades, de tal modo que, después de la I Guerra Mundial, prácticamente todos los grandes bancos trataban, en mayor o menor medida, de participar directamente en la gestión de sus negocios industriales ${ }^{11}$.

Sin embargo, el hecho particular de este período que más ha llamado la atención de los historiadores económicos es la crisis de la banca catalana. La crisis de los bancos catalanes es espectacular: mientras que en 1889 poseían nada menos que el 55,8 de los activos de la banca privada, en 1913 su participación había descendido a sólo el 13,6 por 100. El proceso de crisis de la banca catalana no se detuvo aquí, sino que continuó hasta que el Banco de Barcelona quebró en 1920 y los negocios de la plaza fueron controlados mayoritariamente por entidades con sede en Bilbao o Madrid ${ }^{12}$. Se han esgrimido varias hipótesis para explicar este fracaso de la banca catalana. Por un lado, se encuentran aquellos que achacan a la escasa demanda de servicios financieros el fracaso de la banca catalana ${ }^{13}$. Guillem Graell y Carles Sudrià insisten en la falta de «materia bancable» en Cataluña; es decir, la falta de letras de cambio para descontar en las entidades catalanas. Según estos dos autores, debido a la debilidad y las enormes fluctuaciones del mercado interior, los comerciantes españoles eran incapaces de aceptar órdenes de pago por adelantado y, por ello, los industriales catalanes encontraban enormes dificultades para descontar algún «papel» en los bancos locales. En cambio, Pedro Tedde de Lorca insiste que los principales detonantes de la crisis fueron la propia estructura industrial de Cataluña, con empresas muy pequeñas y poco proclives a recurrir a la financiación bancaria, y la crisis colonial que hizo perder mercados a las empresas catalanas. Por otro lado, Juan Muñoz ha insistido en que fue la propia ineficacia de sus gestores la que llevó a su declive a la banca catalana ${ }^{14}$.

Hay que resaltar que todos los cambios que hemos mencionado con anterioridad se produjeron en un ambiente liberal con una regulación bancaria mínima y sin intervención directa del Estado. De hecho, la legislación bancaria que regía a principios de siglo $\mathrm{xx}$ era bastante permisiva respecto a la creación de nuevas entidades, el permiso a entidades extranjeras para

\footnotetext{
11 Tortella y Palafox (1983).

12 Cabana (1978).

${ }_{13}$ Graell (1908), Tedde de Lorca (1974), y Sudrià (1987).

${ }^{14}$ Muñoz (1988).
} 
establecerse en suelo español y la posibilidad de establecer sucursales ${ }^{15}$. Sin embargo, concedía un enorme poder al Banco de España, que no sólo monopolizaba el derecho de emisión, sino que también podía participar en todo tipo de negocios bancarios ${ }^{16}$. En otras palabras, existía competencia, pero no en términos de igualdad para todos los bancos. Este entorno jurídico no fue modificado hasta la promulgación de la Ley de Ordenación Bancaria de Francesc Cambó en 1921. Esta Ley, que surgió en respuesta a la crisis de las principales entidades bancarias catalanas en 1920, trataba de regular el sistema financiero estableciendo, entre otras medidas, unas condiciones mínimas para la constitución de nuevas entidades financieras. También concedia el carácter de organismo inspector al Consejo Superior Bancario, formado por los mismos bancos privados. De hecho, esta nueva legislación rompía de manera definitiva con un período de relativa liberalidad y hacía una clara apuesta por el intervencionismo estatal ${ }^{17}$. Además, al establecer unos tipos de interés mínimos y otras condiciones similares sobre la captación de recursos ajenos, restringió la competencia entre las diferentes entidades.

\section{METODOLOGÍA}

En esta sección vamos a presentar una explicación muy sucinta, centrada en su interpretación económica, de los métodos que utilizaremos para medir la evolución de la eficiencia a lo largo del artículo, relegando la formulación de los modelos al Apéndice Matemático. El análisis de la productividad relativa de los distintos bancos se ha realizado con índices de productividad calculados mediante el método conocido como DEA ( $\mathrm{Da}$ ta Envelopment Analysis), mientras que su evolución se ha analizado recurriendo al índice de productividad de Malmquist ${ }^{18}$.

El concepto de productividad o eficiencia hace referencia a la economía de medios para obtener unos determinados objetivos, esto es, a la relación entre producto e insumo. Por lo tanto, la medida más natural de productividad es el cociente entre producción y recursos. Un problema clásico al elaborar este cociente es cómo deben ser agregados los diversos pro-

${ }^{15}$ Martín Aceña (1985).

${ }^{16}$ Anes (1974) y Tortella (1970).

17 Martín Aceña (1985) y Pons (1999).

is Caves et al. (1982) proporciona una aproximación general a la metodología de los indices de productividad. 
ductos e insumos. La solución adoptada en el método DEA consiste en medir la eficiencia de forma radial ${ }^{19}$. Es decir, se considera que la productividad se incrementa en un cierto volumen sólo si se incrementan todos los productos simultáneamente en ese mismo volumen sin consumir más insumos, $\mathrm{o}$, alternativamente, que se produce un ahorro de una parte de los recursos sólo si todos los insumos se reducen en esa misma medida sin reducir la producción ${ }^{20}$. En la práctica, para comprobar si se han producido mejoras en productividad se compara cada unidad productiva con la frontera de producción formada por las unidades para las que se observa mejor comportamiento. La figura 1 ilustra la aplicación del modelo CCR (la versión del método DEA que vamos a emplear en este estudio) ${ }^{21}$ al

\section{FIGURA 1}

El método DEA

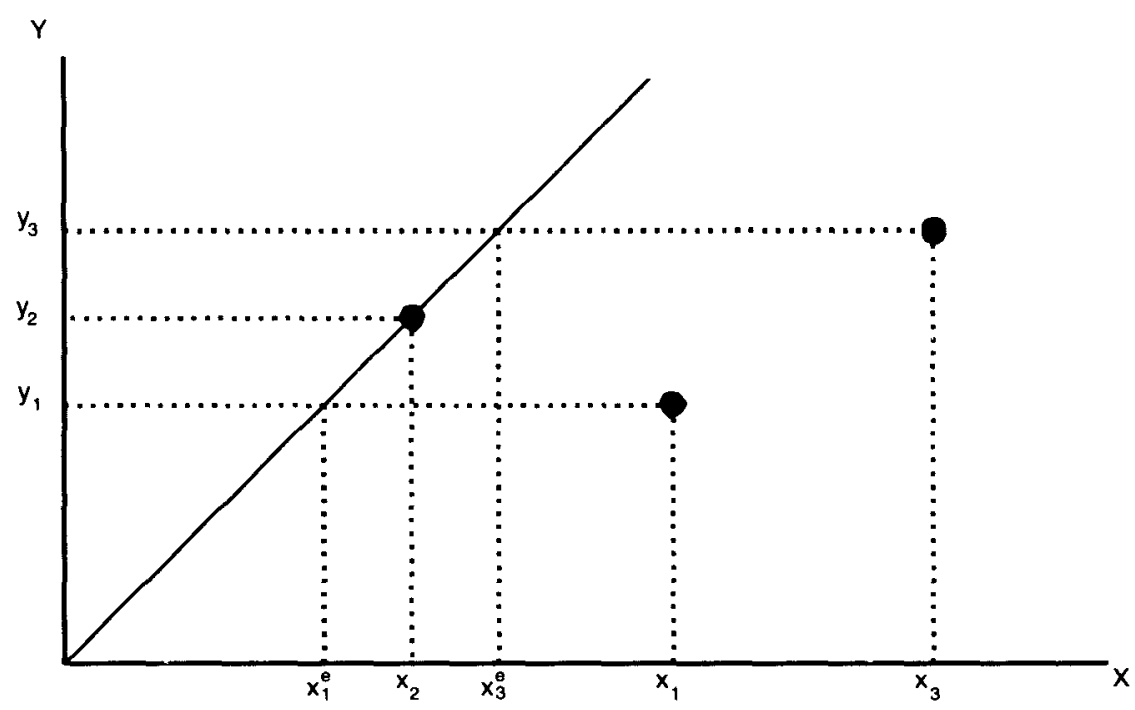

${ }^{19}$ El DEA se presentó en Charnes et al. (1978), tomando como base el concepto de eficiencia de Farrell (1957), y dando lugar a partir de entonces a numerosos desarrollos $\mathrm{y}$ aplicaciones.

${ }^{20}$ Por tanto, no se parte de ningún supuesto sobre la forma de la función de producción y las condiciones del mercado.

${ }^{21}$ En concreto, nos referimos al modelo CCR, que se basa en el trabajo de Charnes et al. (1978), que asume rendimientos constantes a escala. 
caso de un único insumo $(X)$ y un único producto $(Y)$, de modo que cada punto representa los valores correspondientes a una sola unidad productiva.

En la figura anterior, la unidad 2 (definida por la intersección de $\mathrm{Y}_{2}$ y $\mathrm{X}_{2}$ ) es la única eficiente, ya que presenta la razón menor entre insumos y productos, definiendo, por tanto, la frontera eficiente con la que se van a comparar las otras dos unidades. Así, si la productividad de las otras dos unidades fuese igual a la de la unidad 2, mantendrían sus niveles de producto, $y_{1}$ e $y_{3}$, con unos insumos respectivos de $x_{1}^{e}$ y $x_{3}^{e}$, menores que los observados $\left(x_{1}\right.$ y $\left.x_{3}\right)$, por lo que los índices de eficiencia serán $x_{1}^{e} / x_{1}$ y $x_{3}^{e} / x_{3}$. El indice de eficiencia toma un valor unitario para las unidades eficientes (en el ejemplo, la unidad 2) y tiende a cero a medida que éstas son menos eficientes.

La información proporcionada por el método DEA es de carácter estático y, obviamente, en los estudios históricos resulta fundamental ofrecer un análisis temporal. El índice de productividad de Malmquist ${ }^{22}$, que, por otro lado, también se basa en el método anterior, proporciona esa perspectiva dinámica. Para exponer sus fundamentos tomaremos como punto de partida los resultados de aplicar el método DEA, por separado, a los datos de dos períodos, $t$ y $t+1$. En la figura 2 se ilustra esta situación, continuando con el ejemplo de un insumo y un producto, representándose las fronteras eficientes de los dos períodos y las observaciones de una misma unidad en cada uno de ellos.

El Índice de Malmquist entre los períodos $t$ y $t+1$, tomando como referencia la tecnología del periodo $t\left(M_{t, t+1}^{t}\right)$, es el cociente entre el índice de eficiencia de la observación correspondiente al último período, evaluada respecto a la frontera eficiente del primer período $\left(E_{t+1}^{t}\right)$ y el índice de eficiencia de la observación del primer periodo, comparada con la frontera de ese mismo momento $\left(E_{t}^{t}\right)$. Un índice superior a la unidad significa que la productividad ha mejorado del periodo $t$ al $t+1$ mientras que valores menores a uno implican un retroceso en este aspecto. En concreto, en el ejemplo anterior existe una mejora de la productividad, puesto que la observación en $t+1$ tiene una mayor productividad que la de la frontera eficiente inicial.

${ }^{22}$ Respecto a esta metodología, véanse Färe et al. (1985), Färe et al. (1994), Bjurek (1996) y Färe et al. (1997). Entre las aplicaciones de la misma a la banca se encuentran Berg et al. (1992), Berg et al. (1993), Grifell-Tatjé et al. (1996), Pastor (1995) y Wheelock et al. (1999). 
FIGURA 2

El indice de productividad de Malmquist

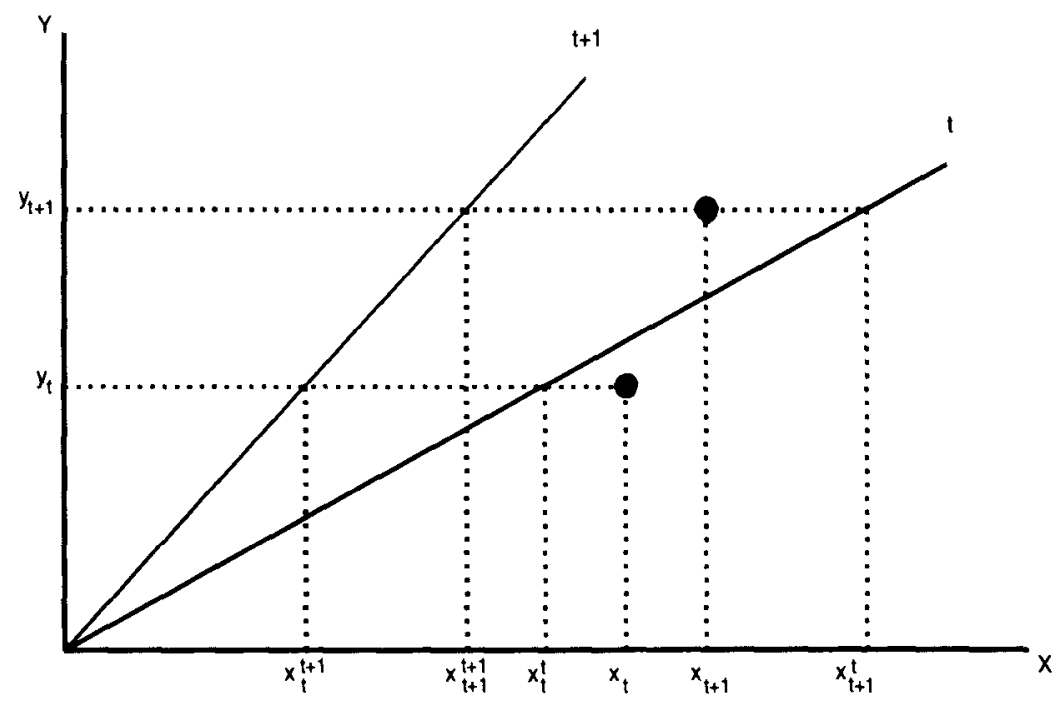

Es posible descomponer la variación de la productividad en dos términos, de modo que el indice anterior sea igual al producto de ambos ${ }^{23}$. El primero recoge la variación debida al desplazamiento de la frontera eficiente, por lo que expresa el grado en que el sector (en la medida en que esté bien representado por las unidades analizadas) ha experimentado un cambio técnico. De manera similar a los casos descritos con anterioridad, valores superiores a la unidad indicarían progreso tecnológico, y valores inferiores, retroceso tecnológico. El segundo (catching-up) expresa la variación achacable a la mejoría del rendimiento relativo de la unidad respecto a las mejores de cada período, esto es, su acercamiento a la frontera eficiente, en caso de que el valor sea mayor que uno, o su alejamiento de la misma, en caso de que sea inferior a la unidad. Estos conceptos pueden ilustrarse haciendo uso de la figura 2. En ella se observa que la mejora en la productividad de la unidad analizada se debe a un importante progreso técnico en el sector, como muestra el importante incremento en la pen-

${ }^{23}$ En el Apéndice se recogen las formulaciones matemáticas del índice y de su descomposición. 
diente de la frontera, y a pesar de que se produce un alejamiento respecto a la frontera eficiente, esto es, de que la unidad analizada empeora su comportamiento respecto a las mejores unidades de cada período.

Una vez descritas las técnicas aplicadas, conviene hacer una reflexión en torno a su utilidad y sus limitaciones. Es importante tener en cuenta que los modelos de frontera utilizados no son sino técnicas de carácter exploratorio que facilitan la interpretación de un conjunto de datos demasiado amplio como para que resulte sencillo obtener conclusiones mediante un análisis no formalizado. Concretamente, en nuestra aplicación analizaremos la evolución de seis variables en dieciséis entidades financieras a lo largo de quince años, lo que proporciona un total de más de 1.400 datos (en realidad, nuestra muestra es algo inferior debido a que no todas las entidades operaron a lo largo de todo el período). En particular, una de las más notables virtudes del método DEA es que permite obtener un índice de eficiencia único a partir de diversas variables indicadoras de su rendimiento y de su consumo de recursos sin necesidad de otorgar a priori unos pesos a las mismas, puesto que el modelo otorgará las ponderaciones más favorables a cada unidad estudiada.

Entre los problemas del método DEA, propios de una técnica determinista de frontera, suele destacarse la sensibilidad de los resultados a la presencia de observaciones extremas. Más concretamente, la presencia de unidades cuya productividad fuese extraordinaria podría provocar grandes distorsiones, puesto que afectaría a las evaluaciones de todas aquellas unidades que se comparasen con ellas. En cambio, la existencia de organizaciones con un rendimiento extremadamente desfavorable, como podría calificarse a alguna de las que hemos estudiado, no tiene ninguna repercusión en la evaluación de las demás y sólo afecta a la significación de su propio índice. Por otro lado, el análisis dinámico, además de proporcionar valiosa información relativa a la evolución de la productividad de las entidades y del sector, ayuda a reducir el impacto de las observaciones anómalamente eficientes, pues cabe esperar que al menos las causas de este problema que tengan un carácter aleatorio (errores de registro o trascripción, circunstancias extraordinarias específicas de una firma, etcétera) sólo tengan incidencia en un número muy limitado de períodos.

Por último, y enlazando con la presentación de los datos que sigue, debe aclararse que el método DEA no ofrece ninguna ayuda en la selección de variables. Los criterios de selección que se han utilizado en el presente estudio no han sido, por tanto, matemáticos o estadísticos, sino que los productos y los insumos se han incorporado a través del análisis económico 
de la función de producción en banca, inspirado en la literatura científica relevante; aunque la elección se ha visto condicionada en parte por la disponibilidad de datos.

\section{LOS DATOS}

Los datos que hemos utilizado en este estudio proceden de las memorias semestrales o anuales de los bancos privados, que en su mayoría se conservan en el archivo del Banco de España en Madrid. Las series de productos (préstamos, cartera, cuentas corrientes, depósitos y cuentas de ahorro) fueron recogidas y estandarizados por Pedro Tedde de Lorca y Gabriel Tortella ${ }^{24}$ y hemos decidido tomarlas directamente de su obra sin ninguna modificación. En cambio, la serie de gastos generales, que han sido utilizados como insumos, ha sido obtenida directamente de las fuentes. La ausencia de datos de la partida de gastos generales en varios bancos nos ha obligado a limitar el tamaño de la muestra a 16 bancos, entre los que se encuentran todas las entidades más importantes. Concretamente, las entidades analizadas son: el Banco de Barcelona (BBCN), el Banco de Sabadell (BSB), el Banco de Valls (BVALLS), Crédito y Docks (CDOC), el Banco de Castilla (BCAST), el Banco Español de Crédito (BEC), el Banco Hispano Americano (BHA), el Banco de Bilbao (BBILBAO), el Banco del Comercio (BCOM), el Banco Guipuzcoano (BGUI), el Banco de Vitoria (BVIT), el Banco de Vizcaya (BVIZ), el Crédito Navarro (CNAV), el Banco Asturiano (BASTIC), el Banco de Santander (BSAN) y el Crédito Balear (CBAL). Por tanto, la muestra comprende cuatro bancos catalanes, tres bancos madrileños, seis bancos vasconavarros y tres radicados en otras regiones, respectivamente, Asturias, Cantabria y las Islas Baleares. Seis de estos bancos fueron creados con anterioridad a 1874, cuatro entre dicha fecha y 1899 y, por último, otros seis a partir de ese año.

Un problema esencial en el análisis de las empresas del sector servicios, en general, y de los bancos, en particular, es la definición de productos e insumos. En este estudio hemos considerado que tanto partidas del activo (préstamos y cartera) ${ }^{25}$ como del pasivo (cuentas corrientes, depósitos y cuentas de ahorro) conforman el producto de los bancos. La razón es que tanto las partidas de activo como las de pasivo se deben de administrar

${ }^{24}$ Tedde de Lorca y Tortella (1974).

25 Debemos notar que la categoría «préstamos» agrupa diversas modalidades (hipotecarios, con garantía, en cuenta corriente, etc.) y que la categoría «cartera» está compuesta 
$y$, por tanto, se pueden considerar como un servicio que el banco presta a sus clientes ${ }^{26}$. Además, tal como es la norma en este tipo de estudios, los datos correspondientes a los productos han sido deflactados por un índice del coste de la vida ${ }^{27}$.

Tal como ya hemos mencionado, la partida de los balances de los bancos conocida como gastos generales, que incluye gastos de personal y de gestión, deflactada por un índice de salarios reales ${ }^{28}$ forma nuestros insumos. Hay que resaltar, además, que gran parte de los gastos generales de los bancos estudiados son gastos de personal. En concreto, en todos los casos para los que disponemos de un desglose de la partida de gastos generales, los gastos de personal suponen entre un 50 y un 70 por 100 de dichos gastos sin que existan diferencias notables entre los diversos bancos. Por tanto, se puede suponer con total confianza que la imposibilidad de desglosar dicha partida no provoca grandes desviaciones en nuestros resultados. Resulta necesario mencionar que la forma ortodoxa de considerar el insumo trabajo en los análisis de productividad a partir de las funciones de producción sería contabilizando el número de trabajadores. No obstante, dada la heterogeneidad que existe entre éstos, la sustitución del simple recuento de empleados por la masa salarial presenta la ventaja de reflejar, de alguna manera, el capital humano presente en la mano de obra. La falta de datos nos ha impedido la incorporación de variables como el activo real o el número de oficinas, que podrían aportar matizaciones a las conclusiones del estudio ${ }^{29}$. Sin embargo, debe tenerse en cuenta que, primero, aunque existan diferencias en la orientación de las entidades que se escapen por esta causa, debe esperarse que se reflejen en el diferente peso relativo de sus productos, y, segundo, debe existir una cierta proporción entre el número de variables y el número de unidades. Si se analizan demasiadas

por el descuento de efectos (letras de cambio y pagarés) y valores (acciones, obligaciones y bonos).

${ }^{26}$ Pastor (1995) y Grifell-Tatjé y Lovell (1996).

27 En este caso, Ballesteros (1997).

${ }^{28}$ Reher y Ballesteros (1993).

${ }^{29}$ En un principio, al contrario de lo que es norma en este tipo de estudios -véase, por ejemplo, Pastor (1995) o Grifell-Tatjé y Lovell (1996)—, pretendíamos incluir como insumo el activo real (principalmente edificios y mobiliario de los mismos), pero las series elaboradas al respecto presentaban importantes anomalías que desaconsejaron su posterior uso. En primer lugar, carecíamos de series completas para tres de los dieciséis bancos analizados. De los trece restantes, en cuatro el activo real decrecía fuertemente a lo largo de los años analizados, sin que esta caída se correspondiera con una disminución de la actividad, según reflejan la evolución de los gastos generales y del producto, y en ocho el activo real se encontraba negativamente correlacionado con dos o más de sus productos. 
variables respecto al tamaño de la muestra de entidades, el método DEA, que toma el punto de vista más favorable a cada una de ellas, le otorgará a la mayoría índices de eficiencia muy elevados pero que difícilmente podrán considerarse verdaderamente representativos de un rendimiento destacado.

\section{LA EVOLUCIÓN DE LA EFICIENCIA DE LOS BANCOS PRIVADOS ESPAÑOLES}

A renglón seguido vamos a estudiar la evolución de la productividad de las entidades de la banca privada que componen nuestra muestra utilizando, en primer lugar, un índice estático de eficiencia que mide la productividad relativa de un banco respecto de los bancos eficientes que tienen una menor relación de insumos por unidad de producto. El índice puede tomar valores entre 0 y 1 , correspondiendo este último a las unidades eficientes. Así, la tabla 1 presenta un índice de eficiencia relativo para los distintos bancos españoles en el año inicial y final de la muestra.

De esta tabla destaca de manera prominente el incremento de la eficiencia media, que crece casi un 35 por 100 del año inicial al final. Este incremento de eficiencia no fue exclusivo de unas pocas entidades, ya que todos los bancos que componen la muestra, excepto Crédito y Docks y el Banco Hispano Americano, lo experimentaron. Además, mientras que en el año inicial sólo cuatro bancos, de dieciséis, se encontraban en la frontera de eficiencia, es decir, su eficiencia relativa era de 1 , en el año final ese número había crecido hasta siete. Por tanto, no es difícil concluir, a partir de dichos resultados, que la productividad de la banca progresó de una forma más que notable en los años que van de la pérdida de Cuba a la I Guerra Mundial.

Los índices estáticos de eficiencia, como los que hemos presentado hasta ahora, no son demasiado eficaces cuando queremos analizar el comportamiento de las distintas empresas a lo largo del tiempo porque comparan, para cada año, la productividad de los bancos con una frontera de eficiencia correspondiente a ese mismo año. Por tanto, no nos proporcionan información respecto de los cambios que puede experimentar dicha frontera como resultado del progreso o retroceso tecnológico. Podría suceder, y veremos algún caso significativo en que fue así, que un banco fuera considerado más eficiente en el período final de la muestra que en el período inicial y, sin embargo, estuviera reduciendo su productividad 


\section{TABLA 1}

Índices de eficiencia de la banca privada española

\begin{tabular}{|c|c|c|}
\hline \multirow{2}{*}{ Bancos } & \multicolumn{2}{|c|}{ Eficiencia } \\
\hline & Inicial & Final \\
\hline 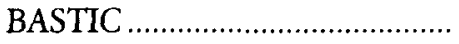 & 0,549 & 0,661 \\
\hline 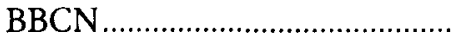 & 1,000 & 1,000 \\
\hline 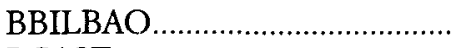 & 0,923 & 0,989 \\
\hline 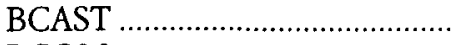 & 0,194 & 0,322 \\
\hline 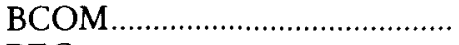 & 1,000 & 1,000 \\
\hline 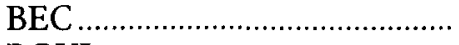 & 0,864 & 1,000 \\
\hline 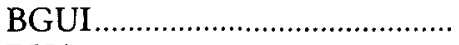 & 0,315 & 0,455 \\
\hline 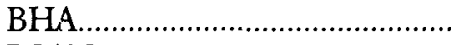 & 0,476 & 0,186 \\
\hline 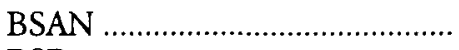 & 0,724 & 1,000 \\
\hline 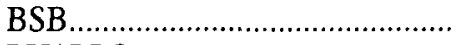 & 0,080 & 0,444 \\
\hline 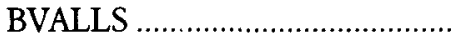 & 0,274 & 0,689 \\
\hline 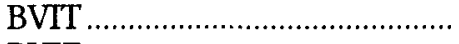 & 0,444 & 1,000 \\
\hline 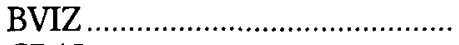 & 0,387 & 0,938 \\
\hline 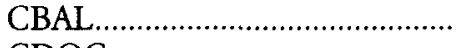 & 1,000 & 1,000 \\
\hline 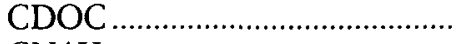 & 0,115 & 0,093 \\
\hline CNAV & 1,000 & 1,000 \\
\hline Media geométrica ....................... & 0,460 & 0,620 \\
\hline
\end{tabular}

NotAS Y FUENTES: En todos los casos el año incial es 1900 excepto en el caso de BBILBAO, BHA, BSB, BVIZ, CDOC que es 1901, en el caso de BVALLS es 1902, en el caso de BEC es 1903, y en el caso de BCAST que es 1906. La fuente de los productos es Tedde de Lorca y Tortella (1974) y la de los insumos las memorias de los bancos.

debido al retroceso tecnológico. El análisis de los índices de Malmquist, que constituyen nuestra principal aportación, nos permitirá resolver este problema y realizar un análisis dinámico de la eficiencia de la banca española durante el período analizado.

La tabla 2 recoge los índices de Malmquist para los distintos bancos que componen nuestra muestra. Valores por encima de la unidad reflejan una mejora de la productividad durante el período, mientras que, por el contrario, valores por debajo de la unidad significan un empeoramiento de la empresa en este aspecto. Hemos calculado dichos índices de dos formas alternativas: primero, comparando de forma directa la situación del año inicial de la muestra con la situación del año final (Modelo 1) $\mathrm{y}$, segundo, examinando la evolución de la productividad para los años 
TABLA 2

Indices de productividad de Malmquist, 1900-1914

\begin{tabular}{|c|c|c|c|c|c|c|}
\hline \multirow{2}{*}{ Banco } & \multicolumn{3}{|c|}{$\begin{array}{l}\text { Evolución de la producctividad del } \\
\text { año inicial a } 1914 \text { (Mod. 1) }\end{array}$} & \multicolumn{3}{|c|}{$\begin{array}{c}\text { Periodos adyacentes: } \\
\text { promedios anuales (Mod. 2) }\end{array}$} \\
\hline & $\begin{array}{l}\text { Catching } \\
\quad-U p\end{array}$ & $\begin{array}{l}\text { Cambio } \\
\text { técnico }\end{array}$ & $\begin{array}{l}\text { Indice de } \\
\text { Malmquist }\end{array}$ & $\begin{array}{l}\text { Catching } \\
\quad \text { Up }\end{array}$ & $\begin{array}{l}\text { Cambio } \\
\text { técnico }\end{array}$ & $\begin{array}{l}\text { Indice de } \\
\text { Malmquist }\end{array}$ \\
\hline BASTIC.. & 1,204 & 0,944 & 1,136 & 1,013 & 0,983 & 0,996 \\
\hline BBCN........ & 1,000 & 0,574 & 0,574 & 1,000 & 0,950 & 0,950 \\
\hline BBILBAO.. & 1,072 & 1,122 & 1,202 & 1,005 & 1,011 & 1,017 \\
\hline BCAST ...... & 1,660 & 0,998 & 1,656 & 1,075 & 0,986 & 1,066 \\
\hline BCOM............... & 1,000 & 1,266 & 1,266 & 1,000 & 0,999 & 0,999 \\
\hline BEC ........ & 1,158 & 0,941 & 1,090 & 1,013 & 0,996 & 1,009 \\
\hline BGUI.. & 1,443 & 0,798 & 1,152 & 1,027 & 1,000 & 1,027 \\
\hline 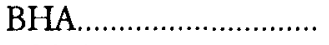 & 0,391 & 0,838 & 0,328 & 0,930 & 0,997 & 0,927 \\
\hline 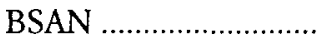 & 1,381 & 1,406 & 1,942 & 1,023 & 0,998 & 1,022 \\
\hline BSB $\ldots \ldots \ldots \ldots \ldots \ldots \ldots \ldots \ldots \ldots \ldots$ & 5,579 & 0,866 & 4,831 & 1,154 & 0,945 & 1,101 \\
\hline BVALLS ... & 2,514 & 0,921 & 2,315 & 1,080 & 0,985 & 1,064 \\
\hline BVIT ....... & 2,252 & 1,604 & 3,613 & 1,064 & 1,030 & 1,093 \\
\hline BVIZ ... & 2,426 & 1,335 & 3,238 & 1,071 & 0,990 & 1,060 \\
\hline CBAL ........................ & 1,000 & 1,650 & 1,650 & 1,000 & 1,030 & 1,020 \\
\hline CDOC & 0,810 & 0,941 & 0,762 & 0,984 & 0,983 & 0,967 \\
\hline CNAV $\ldots \ldots \ldots \ldots \ldots \ldots \ldots$ & 1,000 & 1,296 & 1,296 & 1,000 & 0,995 & 0,995 \\
\hline Media Geométrica....... & 1,349 & 1,055 & 1,422 & 1,026 & 0,992 & 1,018 \\
\hline
\end{tabular}

NOTAS Y FUENTES: En el BBILBAO no hay datos disponibles para 1900, en el BCAST no hay datos disponibles para 1900-1905 y 1908, en el BCOM no hay datos disponibles para 1910, en el BEC no hay datos disponibles para 1900-1902, en el BHA no hay datos disponibles para 1900 , en el BSB no hay datos disponibles para 1901 y 1902, en el BVALLS no hay datos disponibles para 1900 y 1901 , en el BVIT no hay datos disponibles para 1910, en el BVIZ no hay datos disponibles para 1900, en el CBAL no hay datos disponibles para 1907 y 1908 y, finalmente, en el CDOC no hay datos disponibles para 1900. Para las fuentes véase tabla 1 .

adyacentes de la muestra, tomando como variable resumen del período la media geométrica de los índices para cada año (Modelo 2). Cualitativamente, los resultados de ambos modelos son parecidos, aunque obviamente las diferencias de orden absoluto son notables, ya que un modelo muestra el crecimiento acumulado a lo largo de todo el período y el otro modelo el crecimiento anual medio. Un problema del Modelo 1 es que los resultados que se obtienen son muy sensibles a la particular elección 
de los años inicial y final ${ }^{30}$. En ambos casos, también hemos procedido a la descomposición del índice de Malmquist con el fin de diferenciar entre los efectos del cambio técnico y el proceso de catching-up. De manera intuitiva, las mejoras en la productividad de los bancos pueden deberse a dos causas de distinta naturaleza: por un lado, el banco puede estar mejorando su productividad relativa respecto de la frontera eficiente con la que se compara (efecto que llamaremos catching-up) y, por otro, el progreso técnico puede estar desplazando la frontera eficiente que representa la mejor tecnología existente en cada momento del tiempo (efecto que denominaremos cambio técnico).

Observando la tabla 2 constatamos que únicamente tres bancos tienen un índice de productividad de Malmquist significativamente inferiores a la unidad: Crédito y Docks, Banco Hispano Americano y el Banco de Barcelona, que merecerá un comentario detallado más adelante. Según el modelo de períodos adyacentes (Modelo 2), otras tres entidades también empeoran, pero lo hacen a unas tasas insignificantes, de entre el 1 y el 5 por 1.000 anual. A la vista de todo ello, y de los promedios del sector, podemos concluir que se produjo un aumento de los niveles de productividad agregados durante el período estudiado, lo que, obviamente, vendría a refrendar gran parte de lo dicho con los índices estáticos de eficiencia de la tabla 1. El índice de Malmquist del Modelo 1, que compara los años inicial y final, cuantifica el incremento total de productividad en un 42 por 100 , en tanto que el Modelo 2, que tiene en cuenta cada par de años adyacentes, lo estima en un 1,8 por 100 anual.

\section{LAS CAUSAS DE LOS INCREMENTOS DE PRODUCTIVIDAD}

La descomposición del índice de Malmquist de la tabla 2 en catching-up y camibio técnico es una primera aproximación a las causas de los incrementos de productividad. Parece, a todas luces, que el primer efecto fue mucho más importante que el segundo. En otras palabras, el incremento de productividad del sector se debió a una mejora de la eficiencia relativa de las entidades peor situadas respecto a las más avanzadas. Por tanto,

${ }^{30}$ Existe una discusión en la literatura de productividad, véase Jorgenson (1990), sobre la conveniencia de utilizar uno u otro método. En general se tiende a concluir que el mejor resultado se obtiene con la media de la productividad de todos los años que discurren entre los años inicial y final del ciclo económico. Es decir, en nuestro caso, el modelo que mejor se ajusta a este principio es el número 2. 


\section{GRÁFICO 1}

\section{La relación entre tamaño y productividad}

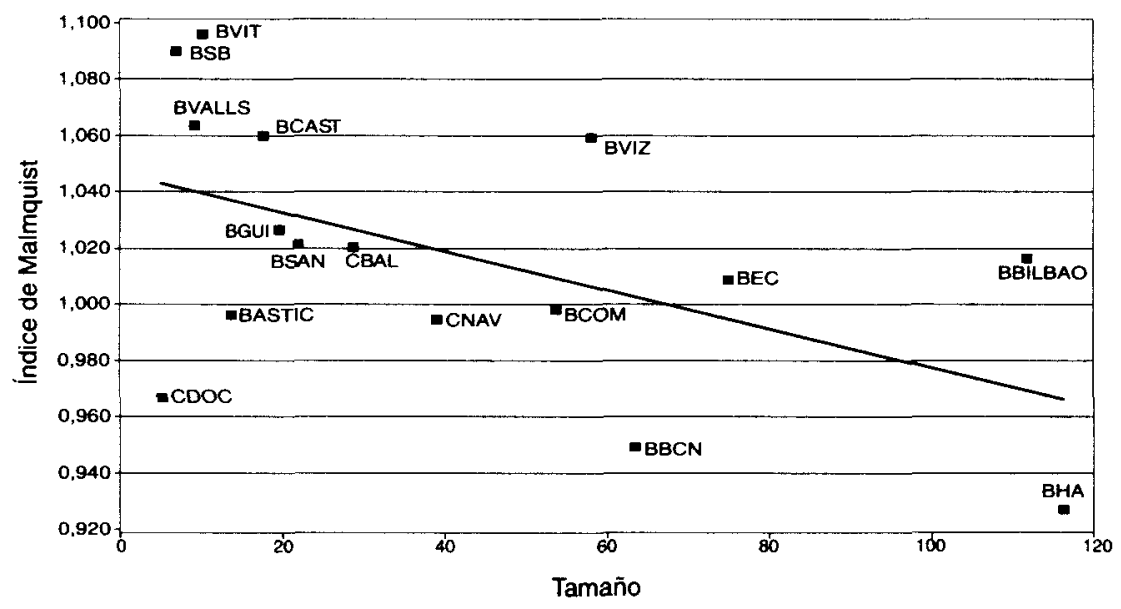

NOTAS y Fuentes: El tamaño se mide como la media aritmética del activo de los bancos a precios constantes a lo largo del período. La fuente de los datos de activo es Tedde de Lorca y Tortella (1974) y el deflactor utilizado procede de Ballesteros (1997). El índice de Malmquist procede de la tabla 2, modelo 2.

el panorama presenta un sector estable, sin grandes innovaciones en los métodos productivos, compuesto por empresas cada vez más expuestas a presiones competitivas, debido al incremento del número de bancos, más que a la extensión de las redes de sucursales, que aumentan sus niveles de eficiencia relativa para poder competir en el mercado ${ }^{31}$.

Sin embargo, existe un proceso de innovación nada desdeñable en siete bancos, tal como muestra el Modelo 1. Además, todos esos bancos son vasconavarros (Banco de Bilbao, Banco del Comercio, Banco de Vitoria, Banco de Vizcaya, Crédito Navarro), excepto dos, el Banco de Santander y el Crédito Balear. Se podría aventurar la hipótesis que este ligero, pero significativo, cambio técnico fue debido a la adopción por parte de estos

${ }^{31}$ Resulta interesante destacar la notable diferencia entre los efectos de este incremento de competencia en la productividad y el que ha tenido lugar en España desde mediados de los años 1980, ya que el más reciente resultó en un descenso de la productividad de las entidades financieras. Véase Grifell-Tatjé y Lovell (1996). 


\section{GRÁFICO 2}

La relación entre tamaño y cambio técnico

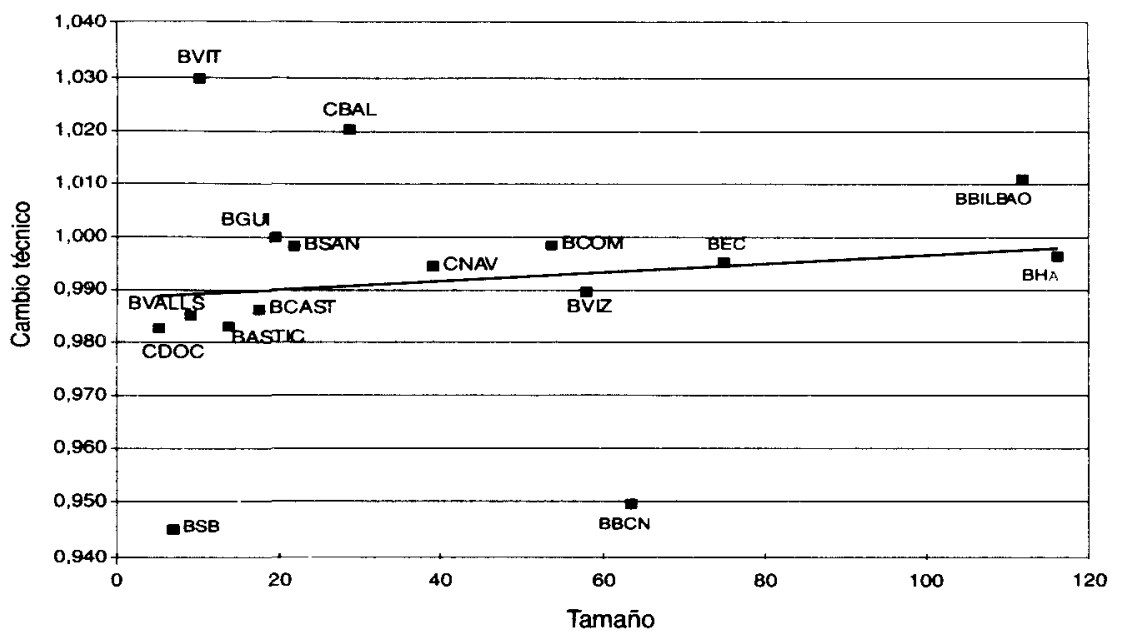

NOTAS y FuENTES: Véase el gráfico 1 para la variable tamaño. El índice de cambio técnico procede de la tabla 2, modelo 2.

bancos de los métodos de gestión de la banca mixta, aunque éste es un tema que merecería un análisis a más largo plazo ${ }^{32}$.

Ahora vamos a ocuparnos de la relación entre el tamaño de los bancos y el crecimiento de la productividad. En concreto, el gráfico 1 ilustra la relación entre los valores del índice de Malmquist y el tamaño de los bancos, medido por el valor medio del total de los activos durante el período. El coeficiente de correlación entre ambas variables es negativo $(-0,523)$, lo que nos indicaría que los bancos más grandes son los que menos han visto aumentar su productividad en el período. De hecho, su inspección detallada nos revela que seis de los siete bancos más grandes presentan un índice de Malmquist que se sitúa por debajo de la media (la única excepción la constituye el Banco de Vizcaya). En otras palabras, parece

32 Existe un largo debate en la literatura internacional sobre si la forma de banca mixta, o universal, era más eficiente que otros tipos de estructuras bancarias. En particular, ha llamado considerablemente la atención la comparación entre la banca alemana (universal) y la banca norteamericana (especializada). Véase al respecto, por ejemplo, Benson (1994), Fohlin (1999) y Kroszner y Rajan (1994). 


\section{GRÁFICO 3}

La relación entre tamaño $y$ catching-up

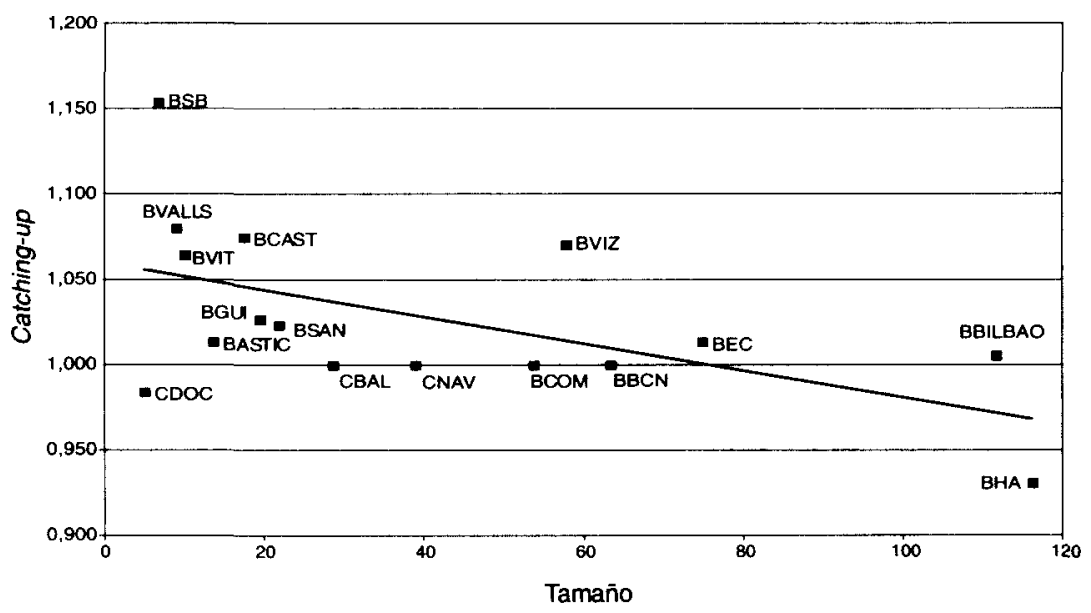

Notas y Fuentes: Véase el gráfico 1 para la variable tamaño. El índice de catching-up procede de la tabla 2 , modelo 2 .

difícil relacionar el incremento de productividad del sector con la presencia de economías de escala entre las grandes instituciones.

Por otra parte, la inspección de los gráficos 2 y 3 revela que los grandes bancos no están muy por debajo de la media en cuanto al cambio técnico (con la importante excepción del Banco de Barcelona), pero, en cambio, sí que se observa que los grandes bancos presentan índices de catching-up muy por debajo de la media. En conclusión, el menor crecimiento de la productividad de los grandes bancos debe relacionarse con su insuficiente catching-up más que con el retroceso tecnológico relativo. Ampliando alguna de nuestras anteriores conclusiones, podríamos afirmar que el panorama bancario que se recoge en nuestra muestra es el de un sector relativamente estable en cuanto a los métodos de producción, pero afectado por presiones competitivas que influyen sobre todo en los bancos más pequeños y que contribuyen a mejorar sus índices de eficiencia relativa. En cierta forma, se puede aseverar que los bancos más pequeñoś son responsables de la mejora de la productividad, gracias a sus progresos en los índices de eficiencia relativa.

Finalmente, puede resultar de gran interés estudiar si el mayor o menor dinamismo productivo de los distintos bancos está conectado con la fecha 
TABLA 3

La influencia de la antigüedad en la eficiencia

\begin{tabular}{|c|c|c|c|c|c|c|}
\hline \multirow{2}{*}{ Año de fundación } & \multicolumn{3}{|c|}{$\begin{array}{l}\text { Evolución de la producctividad del } \\
\text { año inicial a } 1914 \text { (Mod. 1) }\end{array}$} & \multicolumn{3}{|c|}{$\begin{array}{c}\text { Periodos adyacentes: } \\
\text { promedios anuales (Mod. 2) }\end{array}$} \\
\hline & $\begin{array}{l}\text { Catching } \\
-U p\end{array}$ & $\begin{array}{l}\text { Cambio } \\
\text { técnico }\end{array}$ & $\begin{array}{l}\text { Indice de } \\
\text { Malmquist }\end{array}$ & $\begin{array}{l}\text { Catching } \\
-U p\end{array}$ & $\begin{array}{l}\text { Cambio } \\
\text { técnico }\end{array}$ & $\begin{array}{l}\text { Indice de } \\
\text { Malmquist }\end{array}$ \\
\hline & 1,162 & & & 1,0 & & 1,010 \\
\hline $1874-1898 \ldots \ldots \ldots \ldots \ldots \ldots$ & 1,836 & 0,987 & 1,812 & 1,052 & 0,978 & 1,029 \\
\hline Después de $1898 \ldots \ldots \ldots$. & 1,275 & 1,041 & 1,328 & 1,019 & 0,999 & 1,018 \\
\hline
\end{tabular}

NOTAS Y FUENTES: Los datos proceden de la tabla 2. Los bancos creados antes de 1874 son: BBCN, BCAST, BBILBAO, CNAV, BSAN y CBAL. Los bancos creados entre 1874 y 1898 son: BSB, BVALLS, CDOC y BCOM. Finalmente, fueron creados después de 1898 : BEC, BHA, BGUI, BVIT, BVIZ y BASTIC.

de inicio de sus actividades. Por ejemplo, podría pensarse que las nuevas entidades son más dinámicas que las antiguas o, al contrario, en las ventajas de la reputación y la experiencia acumulada por los más veteranos en el mercado. Para estudiar esta posibilidad, dividimos la muestra total en tres grupos, siguiendo criterios temporales ${ }^{33}$, y calculamos la media geométrica del índice de Malmquist y su descomposición para las tres, para comprobar si existen diferencias significativas entre las tres. Los resultados de este ejercicio se presentan en la tabla 3 . Aunque significativas, las diferencias no nos permiten señalar una clara relación entre antigüedad y eficiencia (a la misma conclusión se podría llegar analizando de forma desagregada los resultados para los distintos bancos de cada generación). Los bancos que más progresan en términos de eficiencia fueron aquellos creados en el período intermedio; es decir, entre 1874 y 1898.

\section{LAS CONSECUENCIAS DE LAS MEJORAS DE PRODUCTIVIDAD}

Es interesante observar cuáles pueden ser algunas de las consecuencias de los diferenciales de comportamiento (en términos de mejora de productividad) de las distintas entidades bancarias sobre su crecimiento en

${ }^{33}$ Una de las particularidades de la muestra que se recoge en este trabajo es que se compone de bancos que fueron creados en tres períodos distintos: seis bancos se fundaron con anterioridad a 1874; otros seis bancos se crearon con posterioridad a 1899; y, finalmente, cuatro bancos se crearon entre esos dos años. 


\section{GRÁFICO 4}

La relación entre crecimiento y productividad

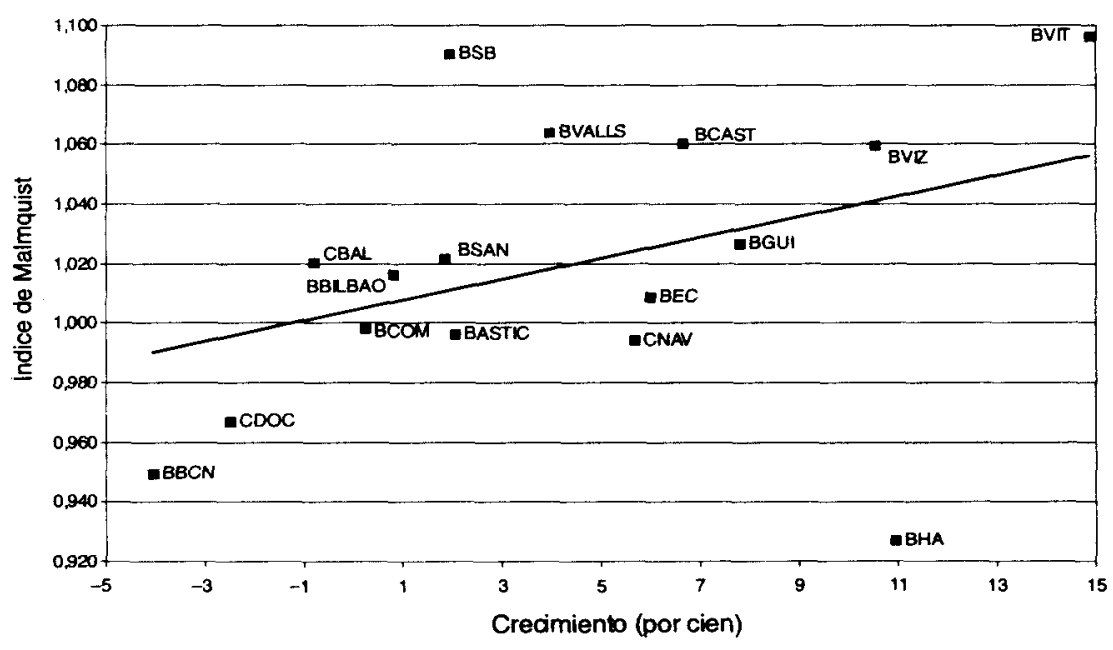

NOTAS Y FuENTES: El crecimiento se mide como la tasa media de crecimiento del activo de los bancos a precios constantes a lo largo del período. La fuente de los datos de activo es Tedde de Lorca y Tortella (1974) y el deflactor utilizado procede de Ballesteros (1997). El índice de Malmquist procede de la tabla 2, modelo 2.

el período. A esto se refieren los gráficos 4,5 y 6 . El gráfico 4 presenta la relación existente entre el crecimiento de las entidades, medido como la tasa media a lo largo del período, y los índices de Malmquist. La inspección del gráfico 4 revela algunos aspectos de interés. En primer lugar, por ejemplo, cuatro de las cinco entidades con mayor crecimiento presentan índices de Malmquist claramente por encima de la media. En segundo lugar, de las cuatro entidades que presentan crecimiento negativo o prácticamente nulo, tres tienen índices de Malmquist claramente inferiores a la media de nuestra muestra, mientras que la restante tiene un valor muy cercano a esa media.

Los gráficos 5 y 6 estudian la relación entre crecimiento y la descomposición del índice de Malmquist entre las variaciones debidas al cambio técnico y las que se deben al proceso de catching-up. La observación del gráfico 5 nos confirma que el cambio técnico fue casi inexistente para una gran parte de las entidades que presentan, por tanto, valores muy 


\section{GRÁFICO 5}

La relación entre crecimiento y cambio técnico

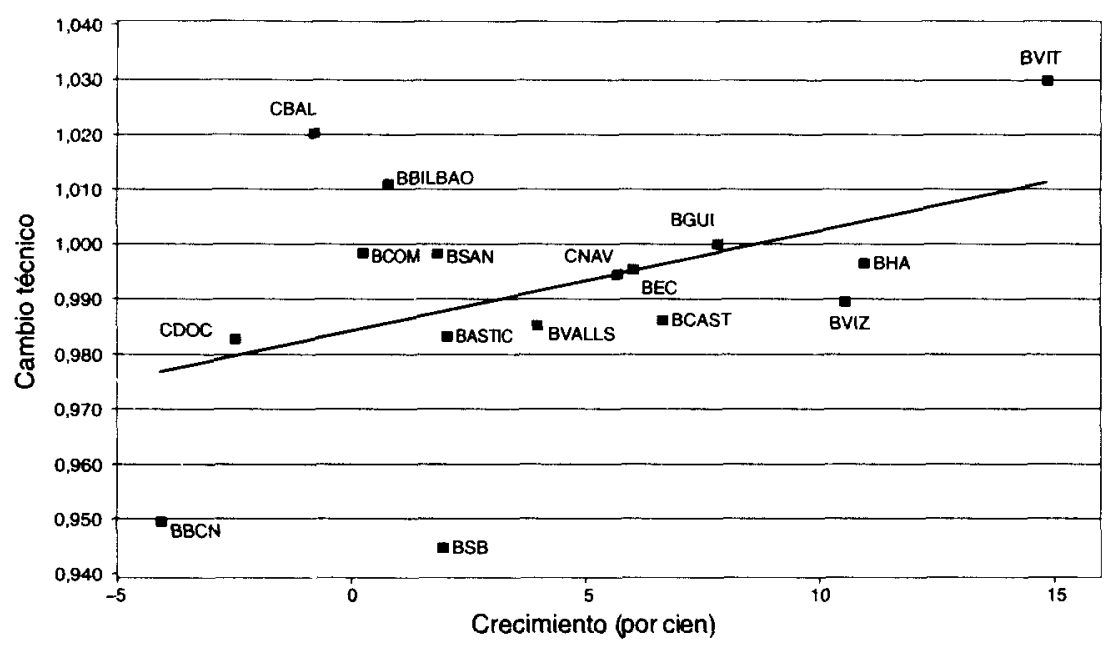

NOTAS Y FuENTES: Véase el gráfico 4 para la variable crecimiento. El índice de cambio técnico procede de la tabla 2, modelo 2 .

cercanos a la unidad. De las dos entidades que presentan progreso técnico sustancial, hay una que presenta el mayor crecimiento del periodo (el Banco de Vitoria) y otra que presenta un comportamiento diametralmente opuesto, incluso con disminución de su actividad (el Crédito Balear). Las entidades que presentan clara regresión técnica tampoco presentan un comportamiento homogéneo, aunque no tan dispar como en el caso anterior. Una de ellas, el Banco de Barcelona, es la entidad que ve disminuir su actividad en mayor medida, mientras que la otra, el Banco de Sabadell, presenta crecimiento positivo, aunque moderado. La regresión técnica del Banco de Barcelona, asociada a la disminución de sus activos, es un anuncio de sus posteriores problemas, que acabaron con su liquidación en el año 1920.

De los ocho bancos que crecen menos durante el período (incluidos aquellos que ven disminuir su tamaño absoluto), siete tienen índices de catching-up claramente inferiores a la media (la única excepción la constituye el Banco de Sabadell), mientras que de los cinco bancos que presentan una mayor expansión durante el período, únicamente uno (el Banco His- 


\section{GRÁFICO 6}

La relación entre crecimiento $y$ catching-up

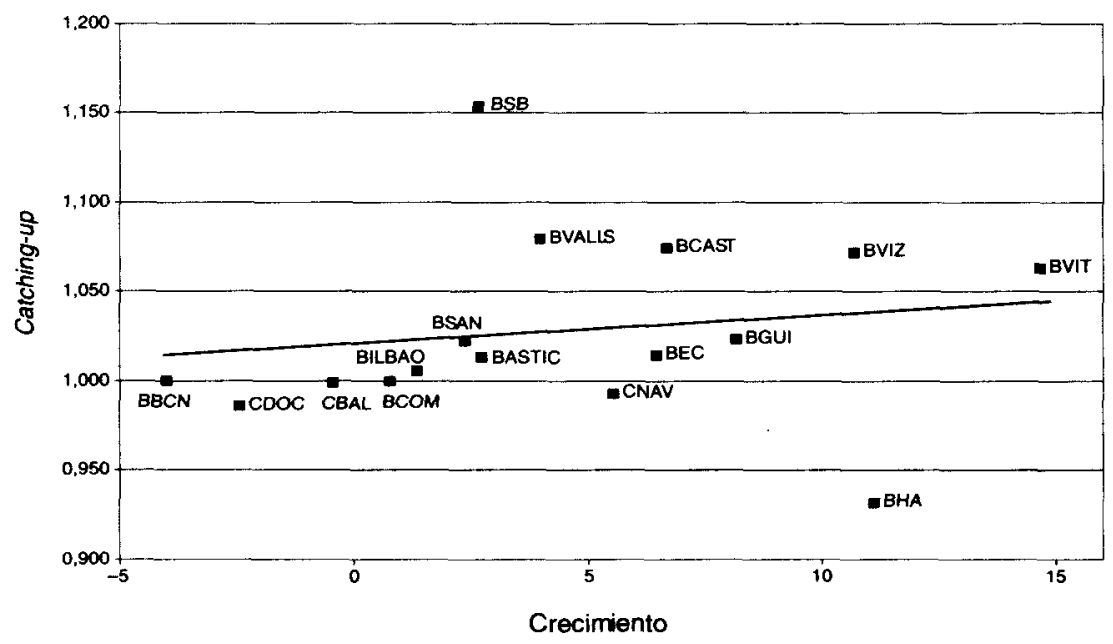

NOTAS Y FUENTES: Véase el gráfico 4 para la variable crecimiento. El índice de catching-up procede de la tabla 2 , modelo 2 .

pano Americano) presenta un índice muy claramente inferior a la media (véase gráfico 6), otro tiene un valor prácticamente igual a la media (el Banco Guipuzcoano), mientras que los otros tres se encuentran claramente por encima. Parece, pues, que puede establecerse una relación entre el proceso de catching-up de los bancos y su crecimiento durante el período. A modo de conclusión podríamos afirmar que parece que existe cierta relación entre el crecimiento de los bancos y su comportamiento en términos de cambios en la productividad. Cambios que, en cualquier caso, se deben más a los distintos procesos de catching-up de las diversas entidades que al cambio técnico que no constituyó un factor importante durante este período.

\section{LA PRODUCTIVIDAD Y LA EVOLUCIÓN DE LOS GRUPOS BANCARIOS REGIONALES EN ESPAÑ̃}

Pasemos ahora a un análisis detallado de la evolución de la productividad de los diferentes grupos regionales en España. La siguiente tabla 4 
TABLA 4

La eficiencia de los grupos bancarios regionales

\begin{tabular}{|c|c|c|c|c|c|c|}
\hline & \multicolumn{3}{|c|}{$\begin{array}{l}\text { Evolución de la producctividad del } \\
\text { año inicial a } 1914 \text { (Mod. 1) }\end{array}$} & \multicolumn{3}{|c|}{$\begin{array}{c}\text { Periodos adyacentes: } \\
\text { promedios anuales (Mod. 2) }\end{array}$} \\
\hline & $\begin{array}{l}\text { Catching } \\
\quad-U_{p}\end{array}$ & $\begin{array}{l}\text { Cambio } \\
\text { técnico }\end{array}$ & $\begin{array}{l}\text { Indice de } \\
\text { Malmquist }\end{array}$ & $\begin{array}{c}\text { Catching } \\
-U_{p}\end{array}$ & $\begin{array}{l}\text { Cambio } \\
\text { técnico }\end{array}$ & $\begin{array}{l}\text { Indice de } \\
\text { Malmquist }\end{array}$ \\
\hline Catalanes ...................... & 1,836 & 0,810 & 1,487 & 1,052 & 0,968 & 1,019 \\
\hline Madrileños...................... & 0,909 & 0,923 & 0,840 & 1,004 & 0,995 & 0,999 \\
\hline Vasconavarros................. & 1,427 & 1,210 & 1,728 & 1,027 & 1,004 & 1,031 \\
\hline Otros.............................. & 1,185 & 1,299 & 1,538 & 1,012 & 1,000 & 1,013 \\
\hline
\end{tabular}

NOTAS Y FUENTES: Los datos proceden de la tabla 2. Los bancos catalanes son: BBCN BBCN, BSB, BVALLS y CDOC. Los madrileños son: BCAST, BEC y BHA. Los vasconavarros son: BBILBAO, BCOM, BGUI, BVIT, BVIZ y CNAV.

nos muestra una media geométrica del comportamiento, en términos de eficiencia, de los diversos bancos que forman nuestras cuatro agrupaciones regionales. El grupo más eficiente, en todos los indicadores, es el vasconavarro, seguido de los bancos catalanes, el grupo de «otros»y, en último lugar, los bancos madrileños. Por lo tanto, a primera vista podría parecer que el éxito de la banca vasconavarra es fácilmente achacable a sus mejoras en eficiencia, incluyendo un notable progreso técnico, mientras que el fracaso de la banca catalana puede difícilmente ligarse a la evolución de su productividad.

Sin embargo, un análisis detallado de las entidades catalanas nos muestra exactamente lo contrario. De las cuatro entidades catalanas analizadas, Banco de Barcelona, Banco de Sabadell, Banco de Valls y Crédito y Docks, tres (todas excepto el Banco de Barcelona) figuran entre las cuatro entidades menos eficientes y lo que realizan a lo largo del período es un proceso de catching-up (tal como precisamente muestra la tabla 4) mientras que experimentan un notable retroceso técnico. Por tanto, todo parece indicar que los problemas del sector financiero catalán tienen su punto de partida en algún momento previo al período analizado y en su especialización productiva, que no permite incrementos de eficiencia con progreso tecnológico. Obviamente, a primera vista, este juicio no debería de ser apropiado para el Banco de Barcelona, que obtiene un índice de eficiencia unitario (véase la tabla 1). Por otro lado, si atendemos a la evolución de la eficiencia a lo largo del período (véase la tabla 2), las trayectorias son bien diferentes: 
mientras Banco de Barcelona y Crédito y Docks figuran entre las tres peores entidades, el Banco de Sabadell tiene la mejor evolución y el Banco de Valls ocupa la cuarta posición en este aspecto. No obstante, debemos resaltar que las posiciones de partida de estos dos últimos bancos son tan precarias que, a pesar de acercarse a la frontera eficiente, no pasan de ocupar, al final del período, las posiciones decimotercera y décima, respectivamente, entre los índices de eficiencia de las dieciséis empresas estudiadas. En esta clasificación, el Crédito y Docks, al sumar a su baja productividad inicial una negativa evolución de ésta, ocupa la última posición, mientras que el Banco de Barcelona obtiene, al igual que ocurría en el período inicial, la calificación de eficiente.

El análisis detallado de la evolución del Banco de Barcelona muestra que, a pesar de que aparezca como eficiente tanto en el primer año como en el último, esta entidad sufre una fuerte crisis durante el período, tal como indica un catching-up que, acumulado, reduce la productividad a aproximadamente la mitad de la inicial. Además, si analizamos individualmente la relación entre el valor medio de cada uno de los componentes del producto (préstamos, cartera, cuentas corrientes, depósitos y cuentas de ahorro) y el valor medio de los gastos generales, se puede constatar que el Banco de Barcelona obtiene buenos resultados, respecto a los recursos utilizados, en sólo en una de las cinco variables analizadas, la correspondiente a cuentas corrientes. Su liderazgo en este aspecto es lo que permite alcanzar los índices de eficiencia unitarios en los períodos inicial y final. En otras palabras, su liderazgo en eficiencia es sólo aparente y probablemente consecuencia de su capacidad para atraer capital en forma de cuentas corrientes. Esta extraordinaria capacidad del Banco de Barcelona podría ser resultado de su dilatada historia que le confería un grado de seguridad y pública confianza de la que no gozaban otras entidades financieras (debe de recordarse que fue fundado en 1844) y de su particular posición de preeminencia dentro del mercado financiero barcelonés ${ }^{34}$.

El gráfico 7 muestra cómo el liderazgo final del Banco de Barcelona se consigue con una productividad que es aproximadamente la mitad del inicial, fenómeno que se oculta en el análisis basado en el DEA por la ausencia de unidades comparables con rendimiento superior al Banco de Barcelona, pero que se explica perfectamente al descomponer los índices de Malmquist. En otras palabras, el Banco de Barcelona perdió a lo largo del periodo capacidad para atraer más capital en cuentas corrientes y no

\footnotetext{
${ }^{34}$ Cabana (1978).
} 


\section{GRÁFICO 7}

Evolución del cociente entre las cuentas corrientes y los gastos generales en el Banco de Barcelona, 1900-1914

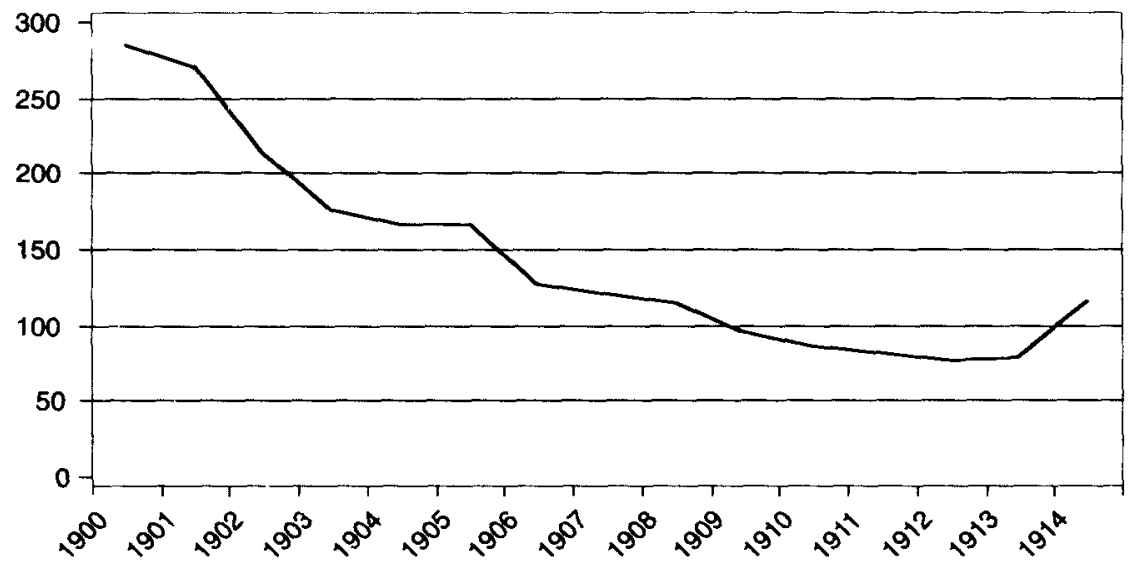

Notas y Fuentes: Memorias del Banco de Barcelona para los años correspondientes.

fue capaz de incrementar su productividad en las otras áreas de negocios. Obviamente, este resultado da cierto soporte a las tesis «endógenas» que ven la crisis financiera catalana como resultado de la falta de dinamismo de las entidades locales.

El comportamiento de la banca madrileña es ciertamente heterogéneo, pese a sus bajos niveles agregados de eficiencia. Mientras que el Banco Español de Crédito tiene un comportamiento positivo en todos los indicadores de productividad (por ejemplo, en nivel de eficiencia pasa del sexto al primer lugar), el Banco de Castilla y el Banco Hispano Americano ocupan en 1914 el antepenúltimo y penúltimo lugar, respectivamente, en niveles de eficiencia. El Banco Español de Crédito nació de la disolución del Crédito Mobiliario Español y gozaba de una fuerte participación de capital francés. Su principal negocio durante este período fueron la bolsa y los servicios financieros a diversas grandes empresas ${ }^{35} \mathrm{y}$, tal como podemos observar, esto le dio bastante buenos resultados al situarse en el período entre la elite de los mejores bancos españoles. El Banco Hispano Ame-

35 Tedde de Lorca (1974), pp. 424-425. 
ricano era el mayor de los tres bancos madrileños aquí estudiados. Se dedicaba a negocios con América Latina que le acarrearon una suspensión de pagos en $1913^{36}$. En cambio, el Banco de Castilla llegó al siglo xx con una cartera formada por negocios industriales y una fuerte vinculación con algunas de las sociedades de Barcelona. La muerte de su fundador, Jaime Girona, en 1907 dio lugar a una reorganización de sus actividades ${ }^{37}$ que, como podemos observar en la tabla 2, sirvió para incrementar su eficiencia absoluta, pero no para mejorar su posición relativa entre los bancos españoles.

El comportamiento de la banca vasconavarra difiere, de manera importante, del de los bancos catalanes y madrileños. Las seis entidades de esa zona consideradas en este estudio experimentaron importantes progresos en el índice de Malmquist durante este período. Un caso espectacular es el del Banco de Vitoria, que pasó del décimo puesto al primero en términos de eficiencia. Hay diversos rasgos de la banca vasca que podrían explicar esa rápida mejora de la eficiencia, aunque a nosotros nos gustaría destacar dos. En primer lugar, la existencia de numerosos nuevos entrantes en el sector, lo que redundó en un aumento generalizado de la competencia que, sin duda, tendría mucho que ver en los incrementos de productividad y en el fenómeno generalizado del catching-up (véase la tabla 2). En segundo lugar, las fuertes diferencias entre la banca vasca y el resto de los bancos españoles en la composición y gestión tanto de activos como pasivos. Así, los bancos vascos tendían a participar directamente en la gestión de sociedades industriales, muchas de nueva tecnología, como las nuevas compañías eléctricas, y a recurrir al pequeño ahorro por medio de libretas de ahorro.

\section{CONCLUSIONES}

El presente estudio pretende profundizar en el análisis de la evolución de la banca privada española en un período, los principios del siglo $\mathrm{xx}$, en el que el sistema financiero se caracterizó por importantes cambios cuantitativos, con un gran crecimiento del número de entidades y del volumen de actividad, y cualitativos, pues dicho crecimiento estuvo acompañado de la modernización de la estructura del sector y de cambios en el peso relativo de los distintos grupos regionales.

36 Tedde de Lorca (1974), pp. 427-430.

37 Tedde de Lorca (1974), pp. 407-408. 
La metodología aplicada, el análisis de la productividad mediante el método DEA (considerando la relación entre los gastos generales de las entidades y sus niveles de actividad, tanto en operaciones de activo como de pasivo), así como el estudio de la evolución de la misma mediante los índices de productividad de Malmquist, permiten extraer interesantes conclusiones que arrojan luz sobre lo acaecido en el referido período.

En primer lugar, destaca el incremento en los niveles de eficiencia en el conjunto del sector. No resulta en absoluto aventurado relacionar esta mejora global con el incremento de la competencia derivado de la creación de numerosas entidades. No obstante, no quiere esto decir que las nuevas organizaciones incorporen una tecnología más eficiente o que su comportamiento sea especialmente dinámico, posibilidades ambas desmentidas por la evidencia empírica obtenida, sino que la consecuencia de la competencia es un acercamiento de las entidades menos eficientes a las más eficientes, en un contexto de estancamiento de la productividad de éstas. Se observa que la mejoría es algo más pronunciada en los bancos de menor tamaño y que generalmente va ligada a unas tasas de crecimiento superiores a la media.

En segundo lugar, el análisis pone de manifiesto el comportamiento diferencial de los distintos grupos bancarios regionales, contrastando especialmente la evolución de la banca catalana y la de la vasconavarra, que explican, al menos parcialmente, el diferente peso que las mismas han venido teniendo desde entonces. Las entidades financieras catalanas parten ya en el período considerado de una posición desfavorable, que se ve agravada por la negativa evolución que sufren a lo largo del mismo, y en especial por el retroceso sufrido por su entidad más emblemática, el Banco de Barcelona (a pesar de que su alto nivel de especialización le sigue otorgando un engañoso cartel de «eficiente» al final del período, que no expresa más que la peculiaridad de su orientación). Por su parte, los bancos vasconavarros experimentan importantes progresos a lo largo de estos años, lo cual, a la vista de la gran cantidad de nuevos entrantes en su ámbito, constituye un argumento más en apoyo de la tesis, anteriormente expuesta, de que la presión competitiva derivada de la creación de nuevas entidades puede considerarse el motor de las mejoras registradas en la productividad.

Finalmente, nos gustaría indicar que este estudio demuestra que el entorno competitivo existente antes de la promulgación de la Ley de ordenación bancaria de 1921 fue altamente beneficioso para el progreso de la industria bancaria en España, puesto que el éxito y el fracaso de los distintos bancos se encontraba ligado a su capacidad para aumentar su 
eficiencia. De hecho, todos los bancos que quebraron después de la I Guerra Mundial son bancos que, precisamente, no destacaban por sus altos niveles de eficiencia.

\section{APÉNDICE MATEMÁTICO}

La formulación matemática del modelo CCR se realiza en términos de programación lineal. En el caso en que existan $n$ unidades productivas, $m$ inputs y soutputs, será:

$$
\begin{gathered}
\min \omega_{0} \\
\text { s.t. } \\
\sum_{j=1}^{n} y_{r j} \lambda_{j} \geq y_{r 0} ; r=1, \ldots, s, \\
\sum_{j=1}^{n} x_{i j} \lambda_{j} \leq \omega_{0} x_{i 0} ; i=1, \ldots, m, \\
\lambda_{j} \geq 0 ; j=1, \ldots, n .
\end{gathered}
$$

donde $x_{i j}$ e $y_{n}$ son las cantidades que la unidad $j$ consume del input $i$ y produce del output $r$, y la unidad analizada se identifica mediante el subíndice 0. Será necesario resolver un planteamiento de programación lineal para calcular el índice de eficiencia de cada unidad.

El modelo puede orientarse a la maximización de los outputs en lugar de a la minimización de inputs, si bien ambos enfoques proporcionan los mismos resultados en tanto se mantenga el supuesto de rendimientos constantes a escala. La equivalencia desaparece si, siguiendo a Banker et al. (1984), se introduce el supuesto de rendimientos variables a escala (modelo $\mathrm{BCC}$ ), añadiendo al planteamiento anterior la restricción

$$
\sum_{j=1}^{n} \lambda_{j}=1
$$

Este cambio permite eliminar del índice de eficiencia del modelo CCR el componente de ineficiencia atribuible a la incorrecta elección de la escala de operación, aunque a costa de un incremento en el número de unidades consideradas eficientes.

En cuanto al Índice de Productividad de Malmquist elaborado a partir de los índices de eficiencia proporcionados por el DEA, tomando como referencia la figura 2, el índice $M_{t, t+1}^{t}$ se calcularía de la siguiente manera: 


$$
M_{t, t+1}^{t}=\frac{E_{t+1}^{t}}{E_{t}^{t}}=\frac{x_{t+1}^{t} / x_{t+1}}{x_{t}^{t} / x_{t}}
$$

La formulación de la descomposición del Índice será:

$$
\begin{gathered}
M_{t, t+1}^{t}=\frac{E_{t+1}^{t}}{E_{t}^{t}}=\frac{E_{t+1}^{t}}{E_{t+1}^{t+1}} \times \frac{E_{t+1}^{t+1}}{E_{t}^{t}}=\begin{array}{c}
\text { Desplazamiento } \\
\text { de la frontera }
\end{array} \times \begin{array}{c}
\text { Acercamiento } \\
\text { a la frontera }
\end{array}= \\
=\left[\frac{x_{t+1}^{t} / x_{t+1}}{x_{t+1}^{t+1} / x_{t+1}} \times \frac{x_{t+1}^{t+1} / x_{t+1}}{x_{t}^{t} / x_{t}}\right]
\end{gathered}
$$

Cuando se incrementa el número de períodos analizados, los resultados pueden perder representatividad debido a la sensibilidad respecto de la elección del año inicial, por lo que ha de modificarse el índice. La modificación más extendida consiste en el cálculo para cada par de períodos adyacentes de un índice que es la media geométrica de dos índices calculados tomando como tecnología de referencia la de cada uno de los períodos, indice que puede desagregarse de forma semejante a la ya expuesta:

$$
\begin{gathered}
M_{t, t+1}^{t, t=\sqrt{\mathrm{M}_{t, t+1}^{t} \times \mathrm{M}_{i, t+1}^{t+1}}}=\sqrt{\frac{E_{t+1}^{t}}{E_{t}^{t}} \times \frac{E_{t+1}^{t+1}}{\mathrm{E}_{t}^{t+1}}}=\frac{E_{t+1}^{t+1}}{E_{t}^{t}} \times \sqrt{\frac{E_{t+1}^{t}}{E_{t+1}^{t+1}} \times \frac{E_{t}^{t}}{E_{t+1}^{t+1}}}= \\
=\begin{array}{c}
\text { Acercamiento } \\
\text { a la frontera }
\end{array} \times \begin{array}{c}
\text { Desplazamiento } \\
\text { de la frontera }
\end{array}
\end{gathered}
$$

\section{BIBLIOGRAFÍA}

Aly, H. Y.; Grabowski, R., Pasurka, C., y Rangan, N. (1990): «Technical, scale and allocative efficiencies in U. S. banking: An empirical investigation», Review of Economics and Statistics, vol. 72, pp. 211-218.

ANES, G. (1974): «El Banco de España (1874-1914): Un banco nacional», en G. Tortella (dir.), La Banca Española en la Restauración (vol. 1), Madrid, Servicio de Estudios del Banco de España, pp. 107-215.

Ballesteros, E. (1997): «Una estimación del coste de la vida en España, 1861-1936», Revista de Historia Económica, vol. 15, núm. 2, pp. 363-395.

Banker, R. D.; Charnes, A., y COOPER, W. W. (1984): «Models for estimation of technical and scale inefficiencies in data envelopment analysis», Management Science, vol. 30, pp. 1078-1092. 
Benston, G. J. (1994): «Universal Banking», Journal of Economic Perspectives, vol. 8, núm. 3, pp. 121-143.

BERG, S. A.; FørSUnd, F. R., y JANSEN, E. S. (1992): «Malmquist indices of productivity growth during the deregulation of Norwegian banking 1980-89», Scandinavian Journal of Economics, vol. 94 (suplemento), pp. S211-S228.

Berg, S. A.; Finn, R. F.; Hjalmarsson, L., y Suominen, M. (1993): «Banking efficiency in the Nordic countries», Journal of Banking and Finance, vol. 17, pp. 371-388.

Berger, A. N.; Hunter, W. C., y Timme, S. G. (1993): «The efficiency of financial institutions: A review of research past, present and future», Joumal of Banking and Finance, vol. 17, pp. 221-249.

BJUREK, H. (1996): «The Malmquist total factor productivity index», Scandinavian Joumal of Economics, vol. 98, núm. 2, pp. 303-313.

CabANa, F. (1978): Història del Banc de Barcelona (1844-1920), Barcelona, Edicions 62.

CANOSA, R. (1945): Un siglo de Banca Privada (1845-1945). Apuntes para la historia de las finanzas españolas, Madrid, Nuevas Gráficas.

CASTANEDA, L., y TAFUNELL, X. (1993): «Un nuevo indicador para la historia financiera española: la cotización de las letras de cambio a corto plazo», Revista de Historia Económica, vol. 11, núm. 2, pp. 367-383.

Caves, D. W.; Christensen, L. R., y Diewert, W. E. (1982): «The economic theory of index numbers and the measurement of input, output and productivity», Econometrica, vol. 50, núm. 6, pp. 1393-1414.

ChARNes, A.; COOPER, W. W., y RHODEs, E. (1978): «Measuring the efficiency of decision making units», European Journal of Operational Research, vol. 2, pp. 429-444.

Färe, R.; Grifell-TatjÉ, E.; Grosskopf, S., y Lovell, C. A. K. (1997): «Biased technical change and the Malmquist productivity index», Scandinavian Joumal of Economics, vol. 99, núm. 1, pp. 119-127.

Färe, R.; GrossKopf, S.; Lindgren, B., y Roos, P. (1994): «Productivity developments in Swedish hospitals: A Malmquist output index approach», en A. Charnes, W. W. CoOper, A. Lewin y L. Seiford (eds.), Data Envelopment Analysis: Theory, Methodology and Applications, Boston, Kluwer Academic Publishers, pp. 253-272.

FÄrE, R.; Grosskopf, S., y LOVelL, C. A. K. (1985): The Measurement of Efficiency of Production, Boston, Kluwer-Nijhoff Publishing.

FARRELL, M. J. (1957): «The measurement of productive efficiency», Journal of the Royal Statistical Society, Series A, vol. 120, pp. 253-281.

FoHLIN, C. (1999): «Universal Banking in Pre-World I Germany: Model or Myth?», Explorations in Economic History, vol. 36, núm. 4, pp. 305-343.

Garcí Ruiz, J. L. (1999): «Bancos Públicos», en P. Martín Aceña y M. Trtos MARTINEZ (eds.), El sistema financiero en España: una sintesis bistórica, Granada, Universidad de Granada, pp. 163-182.

Grabowsk, R.; Rangan, N., y Rezvanian, R. (1994): «The effect of deregulation on the efficiency of U.S. banking firms», Journal of Economics and Business, vol. 46, pp. 39-54. 
GrAell, G. (1908): Sobre la necessitat de crear una banca catalana, Batcelona, Societat d'Estudis Econòmics.

GrIfELl-TATJE, E., y LoVElL, C. A. K. (1996): «Deregulation and productivity decline: The case of Spanish savings banks», European Economic Review, vol. 40, pp. 1281-1303.

JoRGENSON, D. W. (1990): «Productivity and Economic growth», en E. R. BERNDT y J. E. TRIPLETT (eds.), Fifty years of productivity Measurement, Chicago, NBER y University of Chicago Press, pp. 19-118.

Kroszner, R. S., y R. G. Rajan (1994): «Is the Glass-Steagall Act Justified? A Study of the US Experience with Universal banking before 1933, American Economic Review, vol. 84, núm. 4, pp. 810-832.

MaLmQuist, S. (1953): «Index numbers and indifference surfaces», Trabajos de Estadistica, vol. 4, pp. 209-242.

MARTIN ACENA, P. (1985): «Desarrollo y modernización del sistema financiero, 1844-1935», en N. SÁNCHEZ-Albornoz (ed.), La Modernización económica de España, Madrid, Alianza, pp. 121-146.

Millek, S. M., y Noulas, A. G. (1996): «The technical efficiency of large bank production», Joumal of Banking and Finance, vol. 20, pp. 495-509.

MuÑz, J. (1988): El Fracaso de la Burguesía Financiera Catalana (La crisis del Banco de Barcelona), Madrid, Ediciones Endymion.

Pastor Monsalvez, J. M. (1995): «Eficiencia, cambio productivo y cambio técnico en los bancos y cajas de ahorro españolas: Un análisis de frontera no paramétrico», Revista Española de Economia, vol. 12, núm. 1, pp. 35-73.

Pons, M. A. (1999): «La legislación bancaria española», en P. MARTín ACEÑA y M. Trtos Martínez (eds.), El sistema financiero en España: una sintesis histórica, Granada, Universidad de Granada, pp. 35-51.

- (2001a): «Oligopolio y tipos de interés en la banca española, 1942-1975», Revista de Historia Económica, vol. 19, núm. 3, pp. 679-703.

- (2001b): Regulating Spanish Banking, 1939-1975, Oxford, Ashgate.

Rangan, N.; Grabowski, R.; Aly, H. Y., y PasurKa, C. (1988): «The technical efficiency of U.S. banks», Economic Letters, vol. 28, pp. 169-175.

Reher, D., y Ballesteros, E. (1993): «Precios y salarios en Castilla la Nueva: La construcción de un índice de salarios reales», Revista de Historia Económica, vol. 11, núm. 1, pp. 101-151.

Roldán, S., y García Delgado, J. L. (1973): La formación de la sociedad capitalista en España, 1914-1920, Madrid, Confederación de Cajas de Ahorro.

ShePHARD, R. W. (1953): Cost and Production Functions, Princeton, Princeton University Press.

SuDRì̀, C. (1987): «Desarrollo industrial y subdesarrollo bancario en Cataluña», Investigaciones Económicas, vol. 18, pp. 137-176.

TAlLADA, J. M. (1946): Historia de las finanzas españolas en el siglo XIX, Madrid, Espasa Calpe.

TEDDE DE LORCA DE LORCA, P. (1974): «La banca privada española durante la Restauración (1874-1914)», en G. TORTELlA (dir.) La Banca Española en la Restauración (vol. 1), Madrid, Servicio de Estudios del Banco de España, pp. 217-455. 
- (1984): «Banca privada y crecimiento económico en España, 1874-1913», Papeles de Economía Española, vol. 20, pp. 169-183.

Tedde de Lorca de Lorca, P., y Tortella, G. (1974): «Censo y Balances Normalizados de los Bancos Privados Españoles (1874-1914)», en G. TorTella (dir.), La Banca Española en la Restauración (vol. 2), Madrid, Servicio de Estudios del Banco de España.

TrTos, M. (1991): «Las Cajas de Ahorros (1853-1962)», en P. Marín Aceña y M. Trtos MarTínez (eds.), El sistema financiero en España: una sintesis bistórica, Granada, Universidad de Granada, pp. 135-161.

Tortella, G. (1970): «El Banco de España entre 1829 y 1929. La formación de un banco central», en El Banco de España. Una historia económica, Madrid, Banco de España, pp. 261-313.

Tortella, G., y Palafox, J. (1983): «Banca e industria en España, 1918-1936», Investigaciones económicas, vol. 20, pp. 33-64.

WheELOCK, D. C., y WILSON, P. W. (1999): «Technical progress, inefficiency, and productivity change in U. S. banking, 1984-1993», Joumal of Money, Credit and Banking, vol. 31, núm. 2, pp. 212-234.

\section{FUENTES}

Memorias de los siguientes bancos conservadas en la Biblioteca del Banco de España:

Banco Asturiano, 1900-1914.

Banco de Barcelona, 1900-1914.

Banco de Bilbao, 1901-1914.

Banco de Castilla, 1906-1907 y 1910-1914.

Banco de Sabadell, 1900 y 1903-1914.

Banco de Santander, 1900-1914.

Banco de Valls, 1902-1914.

Banco de Vitoria, 1900-1909 y 1911-1914.

Banco de Vizcaya, 1901-1914.

Banco del Comercio, 1900-1909 y 1911-1914.

Banco Español de Crédito, 1903-1914.

Banco Guipuzcoano, 1900-1914.

Banco Hispano Americano, 1901-1914.

Crédito Balear, 1900-1906 y 1909-1914.

Crédito Navarro, 1900-1914.

Crédito y Docks, 1901-1914. 